\title{
Chronic fatigue in patients with unexplained
} self-reported food hypersensitivity and irritable bowel syndrome: validation of a Norwegian translation of the Fatigue Impact Scale

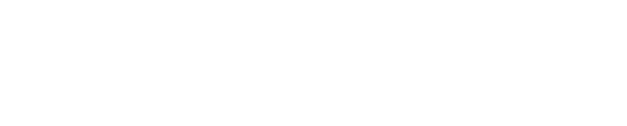

Ragna Lind'

Arnold Berstad ${ }^{2}$

Jan Hatlebakk ${ }^{1,3}$

Jørgen Valeur ${ }^{2}$

'Department of Medicine, Haukeland University Hospital, Bergen, ${ }^{2}$ UngerVetlesen Institute, Department of Medicine, Lovisenberg Diakonale Hospital, Oslo, ${ }^{3}$ Department of Clinical Medicine, University of Bergen, Bergen, Norway
Correspondence: Ragna Lind Department of Medicine, Haukeland University Hospital, N-502I Bergen, Norway

Tel +4755976103

Email ragna.lind@med.uib.no
Background: Patients with unexplained self-reported food hypersensitivity and irritable bowel syndrome (IBS) suffer from several health complaints, including fatigue. The aim of the present study was to validate a Norwegian translation of the Fatigue Impact Scale (FIS), and to assess the impact of fatigue in patients with self-reported food hypersensitivity and IBS, as compared with healthy controls.

Methods: Thirty-eight patients with unexplained self-reported food hypersensitivity and IBS, who participated in the validation of the FIS completed the following additional questionnaires: the Short Form of Nepean Dyspepsia Index for assessment of quality of life, the Subjective Health Complaint Inventory, and questionnaires for diagnosis and severity of IBS. Impact of fatigue was studied in 43 patients with unexplained self-reported food hypersensitivity, 70\% diagnosed with IBS, and 42 healthy controls.

Results: Cronbach's $\alpha$ for the FIS was 0.98 , indicating excellent agreement between individual items. Scores on the FIS correlated with scores on the Short Form of Nepean Dyspepsia Index ( $r=0.50, P=0.001)$, indicating good convergent validity, and were higher in patients (median 85.0, interquartile range 36.8-105.3) than in controls (median 14.0, interquartile range 3.0-29.0, $P \leq 0.0001)$.

Conclusion: The Norwegian translation of the FIS performed excellently in patients with unexplained self-reported food hypersensitivity and IBS, with patients reporting significantly more impact of chronic fatigue than healthy controls.

Keywords: irritable bowel syndrome, fatigue, food hypersensitivity, quality of life

\section{Introduction}

Fatigue may be described as an unusual or extreme sensation of tiredness that is not easily overcome, either by rest or by sleep, and afflicts the whole human being. ${ }^{1}$ When experiences of fatigue are recurrent or persisting for more than 6 months, they are described as chronic. ${ }^{2}$ Fatigue is a common complaint among the general population. Thus, in a cross-sectional population-based study, $22 \%$ of Norwegians reported substantial fatigue, ${ }^{3}$ and in a general medical practice setting, many patients present with unexplained conditions like chronic fatigue syndrome, fibromyalgia syndrome, and irritable bowel syndrome (IBS). ${ }^{4-6}$ In a recent study, patients with IBS reported significantly higher scores on the Fatigue Impact Scale (FIS) compared with healthy controls, even higher than patients with organic gastrointestinal disease. ${ }^{7}$ However, the diagnosis of fatigue is problematic since no biological markers specific to the 
disease have yet been identified, ${ }^{8}$ and there is no generally accepted treatment for the condition. During recent years, studies indicate that low-grade intestinal inflammation may be a contributory factor in the pathogenesis of IBS, as well as in fatigue and musculoskeletal pain conditions. ${ }^{9-11}$ Interestingly, immune-modulating drugs have demonstrated significant clinical efficacy in some patients with severe chronic fatigue. ${ }^{8,12}$

Previous studies in patients with unexplained selfreported food hypersensitivity have shown that the majority suffer from IBS with abdominal pain/discomfort, bloating, and disturbed bowel habits. ${ }^{13,14}$ In addition they had a high prevalence of subjective health complaints, including musculoskeletal pain, ${ }^{15}$ and considerably impaired quality of life. ${ }^{13}$ Sixty-one percent had atopic diseases, ${ }^{16}$ but few, if any, had clear indications of food allergy as a cause of their complaints. ${ }^{17}$ Fatigue was often reported, but not systematically recorded.

Fatigue is usually measured through self-administered questionnaires ${ }^{18}$ which may emphasize different aspects, as severity or impact, of the condition. ${ }^{19}$ The FIS has previously been validated in patients with chronic fatigue syndrome, multiple sclerosis, and liver diseases, ${ }^{19-21}$ and is recommended for clinical use because it has stronger psychometric properties than other fatigue assessment instruments. ${ }^{22}$ The objectives of the present study were to validate a Norwegian version of the FIS, and to study its applicability in patients with unexplained self-reported food hypersensitivity as compared with healthy controls.

\section{Materials and methods}

\section{Participants}

In $2008 / 2009,38$ consecutive adult patients (older than 18 years) with unexplained self-reported food hypersensitivity were examined at Haukeland University Hospital, Bergen, Norway, by an interdisciplinary team, including allergologists, gastroenterologists, psychiatrists, and dieticians, as previously described. ${ }^{14}$ In addition, the patients answered the following questionnaires: the FIS, ${ }^{19}$ the quality of life Short-Form Nepean Dyspepsia Index (SF-NDI), ${ }^{13}$ the Subjective Health Complaints Inventory (SHC), ${ }^{23}$ and two questionnaires concerning IBS, Severity of IBS Symptoms ${ }^{24}$ and Rome III criteria. ${ }^{25}$

During the spring of 2011,70 previously examined patients with unexplained self-reported food hypersensitivity, including the 38 patients from 2008/2009, were telephoned asking if they would take part in a follow-up study by filling out the FIS ${ }^{19}$ and Rome III criteria for IBS. ${ }^{25}$
Fifteen persons did not answer the phone call. Two patients were pregnant or breastfeeding and one had been cured of symptoms, and were therefore excluded. Fifty-two persons agreed to participate and were mailed written information about the study, the questionnaires, and a stamped envelope for return mail. Nine persons did not, after one phone reminder, return the questionnaires. Thus, the results of 43 patients (61\%), remained for analysis, of whom 24 (56\%) had also completed the FIS in 2008/2009. Thirty $(70 \%)$ of the patients fulfilled the Rome III criteria for IBS.

The control group consisted of health care workers, mainly nurses working in shifts at Haukeland University Hospital, who had no contact with the patients. Those who considered themselves to be healthy were given oral and written information about the study. Of the 100 packages of questionnaires that were distributed, 46 were returned. Four (9\%) controls reporting symptoms compatible with IBS were excluded, thus decreasing the control group to 42 participants. Patients and controls provided demographic information like age, gender, profession, and workload. The regional committee for medical research ethics approved the study (REK Vest number 2011/749), and all participants gave written informed consent.

\section{Questionnaires}

\section{Fatigue Impact Scale}

The FIS was originally developed to assess the impact of fatigue on quality of life in patients with chronic diseases. ${ }^{19}$ The questionnaire examines patient perceptions of the functional limitations that they attributed to fatigue during the past month. ${ }^{19}$ The scale is constructed to include three subscales assessing perceived fatigue impact on cognitive functioning (10 items), physical functioning (10 items), and psychosocial functioning (20 items). The statements are ranged on a five-level scale ( $0=$ no problem to $4=$ extreme problems $)$, giving a maximum FIS score of 160 . The FIS is considered a reliable and valid tool to measure the influence of fatigue in different groups of patients. ${ }^{19-21}$

The questionnaire was translated from Swedish ${ }^{20}$ into Norwegian by two of the researchers, and back-translated into Swedish by two translators, one of them being a native speaker of Swedish. The two back-translated and the original versions were then compared and the authors and the translators came to an agreement on the final Norwegian version. Finally, a similar procedure was used for comparing the final Norwegian version with the original English version of the FIS. ${ }^{19}$ 


\section{Short-Form Nepean Dyspepsia Index}

Originally, the NDI consisted of 42 items, measuring healthrelated quality of life structured around 17 key areas of life. ${ }^{26}$ Subsequently, the 10-item SF-NDI was constructed and validated in patients with functional dyspepsia. ${ }^{27} \mathrm{~A}$ validated Norwegian translation of the SF-NDI has previously been found to be a useful tool for the assessment of health-related quality of life in patients with unexplained self-reported food hypersensitivity. ${ }^{13,28}$ The SF-NDI includes five subscales, ie, tension, interference with daily activities, eating/drinking, knowledge control, and work/study. Each subscale contains two items which are measured by a five-point graded Likert scale $(1=$ not at all, $2=$ a little, $3=$ moderately, $4=$ quite $a$ lot, 5 = extremely). A total sum score for quality of life and a sum score for each of the five subscales were calculated by adding up scores for each item (range of total quality of life 10-50, and of each subscale 2-10). Higher scores indicate worse functioning or more severe symptoms.

\section{Subjective health complaint}

The SHC measures subjective, somatic, and psychologic complaints (29 items) experienced during the last 30 days. ${ }^{23}$ The questionnaire has been tested in different populations and has satisfactory validity and reliability. ${ }^{29}$ The questionnaire is divided into five subgroups: musculoskeletal pain (headache, migraine, neck pain, arm pain, shoulder pain, upper back pain, low back pain, and leg pain), pseudoneurology (tiredness, sleep problems, anxiety, sadness/depression, extra heartbeats, heat flushes, and dizziness), gastrointestinal problems (gas discomfort, abdominal discomfort, diarrhea, constipation, gastritis/ulcer, heartburn, and stomach pain), allergy (allergies, breathing difficulties, eczema, and asthma), and influenza (cold/influenza and coughing). The severity of each complaint is rated on a four-point scale $(0=$ not at all, $1=\mathrm{a}$ little, 2 = some, $3=$ severe) .

\section{Rome III criteria and IBS symptom questionnaire}

Based on Rome III criteria, ${ }^{25}$ we used a questionnaire consisting of five questions to diagnose IBS and five questions to describe symptoms. The Rome III criteria was translated from English into Norwegian by the researchers, and back to English by two persons, one of whom had English as her native language. The Norwegian version was made after agreement on minor adjustments between the researchers and translators. The presence of IBS was defined as abdominal pain or discomfort at least 3 days per month during the last 3 months, for at least 6 months, associated with two or more of the following three symptoms: change in frequency of stool, change in form (appearance) of stool, and improvement in defecation. ${ }^{25}$ In addition, the subjects completed a questionnaire (Severity of IBS Symptoms) assessing daily severity of symptoms like nausea, bloating, abdominal pain, constipation, diarrhea, and anorexia. Severity of symptoms is graded on a scale from 0 to 10 , giving each subject a total score of between 0 and $60 .^{24}$

\section{Statistical analysis}

The data were analyzed using GraphPad Prism version 5 (GraphPad Software Inc, San Diego, CA, USA) and Statistical Package for the Social Sciences version 17.0 software (SPSS Inc, Chicago, IL, USA). Validation of the FIS included scale reliability estimated by Cronbach's $\alpha$ coefficient, which ranges from 0 to 1 . An acceptable Cronbach's $\alpha$ has been identified in the literature as $>0.7 .{ }^{30}$ Reproducibility was determined by correlation of the FIS subscores in the same patients $(n=24)$ answering the FIS in 2008/2009 and again in 2011. In this study, we used face validity, which was assessed by having "experts" review the contents of the questionnaire, to see if the items seemed appropriate; convergent validity, which is the degree to which the questionnaire is similar to another questionnaire that it theoretically should be similar to; ${ }^{31}$ construct validity by correlating scores on the SF-NDI, SHC, and Severity of IBS Symptoms questionnaire with scores on the FIS; and known-groups validity, which is determined by the degree to which an instrument can demonstrate different scores for groups known to vary on the variables being measured. ${ }^{31}$ Finally, multiple regression analysis was used to determine predictive factors for fatigue and quality of life.

Because the questionnaires are graded on an ordinal level, nonparametric statistical methods were used. The descriptive statistics used were numbers (n) and percentages (\%), means and standard deviations, and medians and interquartile ranges (IQR). The statistical significance of differences were analyzed using the unpaired Student's $t$-test or Mann-Whitney rank-sum test, Wilcoxon signed-rank test, and odds ratios with $95 \%$ confidence intervals. Correlations were computed using the Spearman rank order correlation coefficient, $r$. Proportions were compared using Fisher's Exact test, and $P<0.05$ was considered to be statistically significant. Too few men precluded a statistical evaluation of the influence of gender.

\section{Results}

\section{Validation of Norwegian version of FIS}

The mean age of the 38 patients in the validation study was $36.1 \pm 15.1$ years, and $30(79 \%)$ were women. The median 
IBS symptom score was 25.5 (IQR 18.8-34.0). The results of the questionnaires are shown in Table 1 . The most frequently reported subjective health complaints were abdominal bloating and tiredness $(89 \%)$ followed by diarrhea $(82 \%)$ and stomach pain $(74 \%)$.

\section{Validity}

Face validity was satisfactory. The total FIS score correlated significantly with quality of life, SF-NDI, and SHC ( $r=0.50$, $P=0.001$ and $r=0.42, P=0.009$, respectively) but not with IBS symptom scores $(r=0.32, P=0.053)$. FIS discriminated significantly between groups of patients; those reporting SF-NDI scores $\geq 27(\mathrm{n}=23$ ) perceived significantly more fatigue (median 92.0, IQR 64.0-109.0) than those with SF-NDI scores $<27$ ( $\mathrm{n}=15$, median 54.0, IQR 9.0-98.0, $P=0.039)$.

\section{Reliability}

Cronbach's $\alpha$ was 0.98 for the total FIS, 0.92 in the cognitive and physical function subscales, and 0.95 for psychosocial function $(n=38)$. There was no significant difference in either total FIS scores or in cognitive, physical, or psychosocial function in the same 24 patients in 2008/2009 and 2011 (Table 2).

Table I Total and subgroup scores expressed as median and interquartile ranges for the Fatigue Impact Scale, Short-Form Nepean Dyspepsia Index, and subjective health complaints questionnaires in patients with self-reported food hypersensitivity and irritable bowel syndrome $(n=38)$

\begin{tabular}{ll}
\hline & Median (IQR) \\
\hline Fatigue Impact Scale & \\
Total score & $88.5(47.8-104.3)$ \\
Cognitive function & $18.5(10.0-29.0)$ \\
Physical function & $24.0($ II.8-30.3) \\
Psychosocial function & $38.5($ I6.3-49.0) \\
Quality of life (SF-NDI) & \\
Total score & $27.5(24.0-37.0)$ \\
Tension & $5.0(4.0-8.0)$ \\
Interference with daily activities & $6.0(4.0-7.3)$ \\
Eating/drinking & $7.0(5.0-9.2)$ \\
Knowledge control & $4.0(3.0-6.0)$ \\
Work/study & $6.0(2.8-8.0)$ \\
Subjective health complaints & \\
Total score & $23.0(14.8-33.5)$ \\
Musculoskeletal pain & $5.5(2.0-11.0)$ \\
Pseudoneurology & $5.0(2.0-10.0)$ \\
Gastrointestinal problems & $7.0(5.0-11.2)$ \\
Allergy & $2.0(1.0-4.3)$ \\
Influenza & $1.0(0.0-2.3)$ \\
\hline
\end{tabular}

Abbreviations: IQR, interquartile range; SF-NDI, Short-Form Nepean Dyspepsia Index.

\section{Multiple regression analysis}

A model including FIS as the dependent variable and quality of life, subjective health complaints, IBS symptoms, and age as independent variables explained $40 \%$ of the variance $\left(r^{2}=0.40, \mathrm{~F}=5.62, P=0.001\right)$ in fatigue where quality of life was the sole significant predictor $(P=0.006)$.

\section{Comparison of FIS in patients and controls}

There were no significant differences in gender or mean age between patients and controls, but significantly more patients were younger than 40 years of age and not employed full-time (Table 3). Cronbach's $\alpha$ value for total FIS among the controls was 0.97 and in the subgroups of cognitive, physical, and psychosocial functions was $0.93,0.90$, and 0.94 , respectively.

Overall, $43(100 \%)$ of the patients reported some impact of fatigue compared with $34(81 \%)$ of the controls $(P=0.003)$. The patients reported significantly $(P \leq 0.0001)$ median higher scores on the FIS (85.0, IQR 36.8-105.3) compared with controls (14.0, IQR 3.0-29.0).

Each domain of the FIS showed significantly higher scores in patients compared with controls, with similar statistically significant differences in all domains $(P<0.0001$, Figure 1). There were no significant differences with regard to impact of fatigue between patients with and without IBS or between patients younger and older than 40 years. The four most marked differences between patients and controls [odds ratio, 95\% confidence interval] were in the five statements: "Normal day-to-day events are stressful for me" [14.6 (5.0-42.8)], "I am not able to provide as much emotional support to my family as I should" [12.1 (4.3-33.6)], "I have to reduce my workload and responsibilities" [10.7 (3.9-29.1)], and "I am less able to complete tasks that require physical effort" [9.8 (2.4-17.2)]. In the patient group, one person did not answer the statement "I am less able to provide financial support for myself and my family", and another did not answer the statement "I engage in less sexual activity" in the FIS. The controls answered all questions.

\section{Discussion}

The results of the present study confirm the validity and reliability of the Norwegian version of the FIS by demonstrating high internal consistency and an excellent correlation between the FIS and measures of quality of life and various subjective health complaints. The ability of the FIS to discriminate different levels of fatigue between patients and controls supports the construct validity of the scale, in 
Table 2 Impact of fatigue in patients with self-reported food hypersensitivity and irritable bowel syndrome in 2008/9 and 20II ( $\mathrm{n}=24)$

\begin{tabular}{|c|c|c|c|c|}
\hline \multirow[t]{2}{*}{ Fatigue Impact Scale } & \multicolumn{2}{|l|}{ Median (IQR) } & \multirow{2}{*}{$\begin{array}{l}\text { Wilcoxon matched-pair } \\
\text { signed-rank test }\end{array}$} & \multirow{2}{*}{$\begin{array}{l}\text { Spearman correlation } \\
\text { coefficient, } r\end{array}$} \\
\hline & $2008 / 2009$ & 2011 & & \\
\hline Total score & $91.0(23.8-108.5)$ & $85.0(|8.5-1| 4.0)$ & $P=0.605$ & $r=0.67, P=0.0002$ \\
\hline Cognitive & $20.5(10.5-28.8)$ & $20.0(5.3-27.5)$ & $P=0.346$ & $r=0.42, P=0.020$ \\
\hline Physical & $24.0(7.3-28.8)$ & $25.5(5.3-34.0)$ & $P=0.457$ & $r=0.57, P=0.013$ \\
\hline Psychosocial & $39.5(17.5-53.5)$ & $4 I .5(8.8-54.2)$ & $P=1.000$ & $r=0.42, P=0.061$ \\
\hline
\end{tabular}

Abbreviation: IQR, interquartile range.

accordance with the results reported by Fisk et al. ${ }^{19}$ High stability of scores over a time interval of about 2 years indicates good reproducibility of the FIS and that fatigue is a persistent condition in our patients with unexplained self-reported food hypersensitivity. The fact that all but two questions were answered by the participants indicates that the Norwegian translation of the FIS was found to be understandable, meaningful, and easy to answer. One may argue that the present study does not support Norwegian validation of the FIS according to gender. However, we believe that our patient sample, with most participants being women, is still representative of the patient population affected by food hypersensitivity and IBS. ${ }^{32}$

Although chronic fatigue syndrome is a common comorbidity in patients with IBS, ${ }^{5}$ there are few studies comparing fatigue in patients with and healthy controls. Consistent with prior results in IBS, ${ }^{7}$ our patients with unexplained selfreported food hypersensitivity and IBS perceived a greater impact of fatigue than healthy controls. Butt et al reported that patients with chronic fatigue syndrome and IBS claimed more severe fatigue than patients with chronic fatigue syndrome without IBS. ${ }^{33}$ In the present study, IBS did not predict fatigue, while quality of life did. However, the SF-NDI and FIS questionnaires include related questions which may in part explain this apparent prediction.

Table 3 Demographic data (n, \%) for patients with self-reported food hypersensitivity and healthy controls

\begin{tabular}{llll}
\hline & $\begin{array}{l}\text { Patients } \\
(\mathbf{n}=\mathbf{4 3})\end{array}$ & $\begin{array}{l}\text { Controls } \\
(\mathbf{n}=\mathbf{4 2})\end{array}$ & P-value \\
\hline Female gender & $38(88)$ & $4 \mathrm{I}(98)$ & 0.202 \\
Age, years (mean, SD) & $4 \mathrm{I}(\mathrm{I} 6)$ & $47(\mathrm{I})$ & 0.052 \\
Age $<40$ years (n, \%) & $24(56)$ & $12(29)$ & 0.015 \\
Employment status (n, \%) & & & \\
$\quad$ Full-time (I00\%) & $14(33)$ & $33(79)$ & $<0.000$ I \\
$\quad$ Part-time (20\%-80\%) & $7(16)$ & $9(2 \mathrm{I})$ & 0.589 \\
Disability pension & $8(19)$ & & \\
Students & $6(14)$ & & \\
Pensioners & $5(11)$ & & \\
Unemployed & $2(5)$ & & \\
Full-time homemaker & $\mathrm{I}(2)$ & & \\
\hline
\end{tabular}

Being unable to provide sufficient financial support for themselves and their family and being in a position of dependence on others were dominating worries among the patients. This is in accordance with the results of a study using the FIS in patients with chronic hepatitis $\mathrm{C} .{ }^{34}$ The results are thus related to the patients' self-reported employment status. When excluding students, $49 \%$ of the patients were employed, and only $33 \%$ worked full-time. It is a serious personal and social problem when young persons (nearly $60 \%$ of the patients were under 40 years) are not capable of maintaining themselves financially. There were some differences between the patient and control groups with respect to age and job. On the other hand, our previous studies do not suggest that the patients' complaints are age and job dependent. ${ }^{35}$ Unexplained somatic symptoms like fatigue and other extraintestinal complaints represent a serious loss, not only for patients, but also for society and health care services in general. ${ }^{36,37}$

The patients self-reported having multiple symptoms from different organ systems including fatigue and food hypersensitivity. Most of the patients were diagnosed with IBS, but none with food allergy. ${ }^{14}$ One cannot exclude the possibility that the results are due to a general tendency to

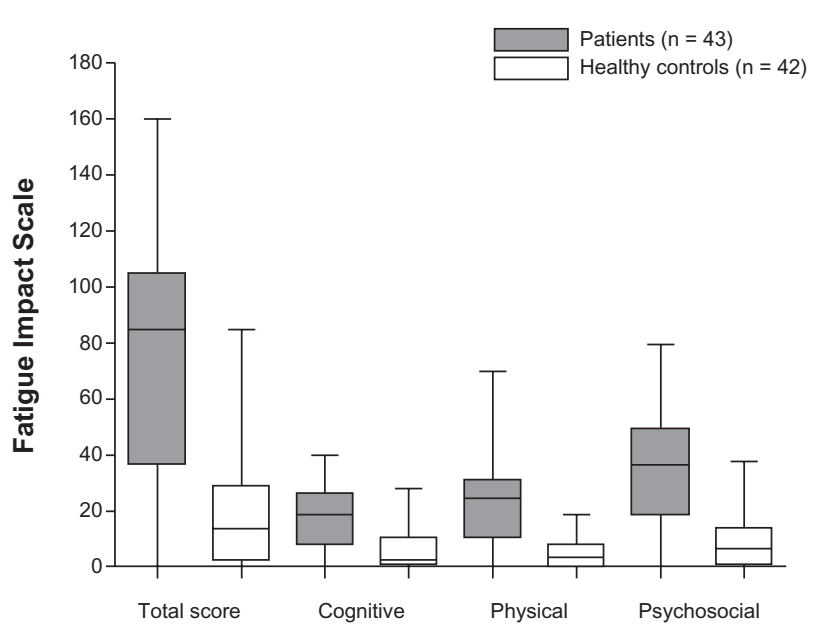

Figure I Total and subgroup scores (medians and interquartile ranges) for impact of fatigue in patients with unexplained self-reported food hypersensitivity versus healthy controls $(P<0.000 \mathrm{I})$. 
amplify bodily sensations in the patient group. However, previous studies using objective methods to assess similar symptoms do not support this view. ${ }^{38,39}$ Patients with unexplained self-reported food hypersensitivity are also particularly responsive to certain fermentable, short-chain carbohydrates, ${ }^{40,41}$ supporting the indication that their food hypersensitivity is indeed a specific feature.

Among employees with heavy workloads and shift works, of which nurses are typical, feeling tired and fatigued are common complaints. ${ }^{42}$ Nurses under the age of 40 years working in shifts are more likely to suffer from sleep disturbances, menstrual irregularities, and adverse pregnancy outcomes than those working in the daytime. ${ }^{42,43}$ Even though health care workers may be prone to underreporting their symptoms, especially in response to questionnaires, ${ }^{44} 83 \%$ of the nurse controls reported some impact of fatigue, which is in accordance with results from otherwise healthy persons. ${ }^{20,34,45}$

A common etiology of multiple unexplained symptoms is an interesting possibility. Indeed, a growing body of evidence indicates similarities in the pathogenesis of chronic fatigue syndrome and IBS, such as alterations in gut permeability, disturbances of gut microbial community, and dysfunctions of immune regulation. ${ }^{46,47}$ The gut microbiota may influence the nervous, endocrine, and immune systems, and also interact with higher brain centers. ${ }^{46}$ Whether such mechanisms play a pathophysiologic role in patients with unexplained self-reported food hypersensitivity certainly warrants further investigation.

In summary, the results of the present study confirm that the Norwegian version of the FIS is a suitable tool for assessment of fatigue in patients with unexplained self-reported food hypersensitivity and IBS, who report considerably more chronic fatigue than healthy controls.

\section{Acknowledgment}

The corresponding author has been supported by grants from the University of Bergen, Bergen, Norway.

\section{Author contributions}

RL and $\mathrm{AB}$ designed the methods and experiments, analyzed the data, interpreted the results, and wrote the paper. JH and JV discussed the analyses, interpretation, and presentation. All authors have contributed to, seen, and approved the manuscript.

\section{Disclosure}

The authors declare no commercial or financial conflict of interest in this work.

\section{References}

1. Swain M. Fatigue in chronic disease. Clin Sci. 2000;99:1-8.

2. Fukuda K, Straus SE, Hickie I, Sharpe MC, Dobbins JG, Komaroff A. The chronic fatigue syndrome: a comprehensive approach to its definition and study. Ann Intern Med. 1994;121:953-959.

3. Loge JH, Ekeberg Ø, Kaasa S. Fatigue in the general Norwegian population: normative data and associations. J Psychosom Res. 1998;45: $53-65$.

4. Bates DW, Schmitt W, Buchwald D, et al. Prevalence of fatigue and chronic fatigue syndrome in a primary care practice. Arch Intern Med. 1993;153:2759-2765.

5. Dansie EJ, Furberg H, Afari N, et al. Conditions comorbid with chronic fatigue in a population-based sample. Psychosomatics. 2012;53: $44-50$.

6. Sperber AD, Atzmon Y, Neumann L, et al. Fibromyalgia in the irritable bowel syndrome: studies of prevalence and clinical implications. Am J Gastroenterol. 1999;94:3541-3546.

7. Simrèn M, Svedlund J, Posserud I, Bjornsson ES, Abrahamsson H. Predictors of subjective fatigue in chronic gastrointestinal disease. Aliment Pharmacol Ther. 2008;28:638-647.

8. Fluge O, Bruland O, Risa K et al. Benefit from B-lymphocyte depletion using the anti-CD20 antibody rituximab in chronic fatigue syndrome. A double-blind and placebo-controlled study. PLOS One. 2011;6:e26358.

9. Akiho H, Ihara E, Nakamura K. Low-grade inflammation plays a pivotal role in gastrointestinal dysfunction in irritable bowel syndrome. World J Gastrointest Pathophysiol. 2010;1:97-105.

10. Ford AC, Talley NJ. Mucosal inflammation as a potential etiological factor in irritable bowel syndrome: a systematic review. $J$ Gastroenterol. 2011;46:421-431.

11. Spiller RC. Postinfectious irritable bowel syndrome. Gastroenterology. 2003;124:1662-1671.

12. Norheim KB, Harboe E, Gøransson LG, Omdal R. Interleukin-1 inhibition and fatigue in primary Sjögren's syndrome - a double blind, randomised clinical trial. PLoS One. 2012;7:1-7.

13. Arslan G, Lind R, Olafsson S, Florvaag E, Berstad A. Quality of life in patients with subjective food hypersensitivity: applicability of the 10-item short form of the Nepean Dyspepsia Index. Dig Dis Sci. 2004;49:680-687.

14. Lind R, Lied GA, Lillestøl K, Valeur J, Berstad A. Do psychological factors predict symptom severity in patients with subjective food hypersensitivity? Scand J Gastroenterol. 2010;45:835-843.

15. Lind R, Arslan G, Eriksen HR, et al. Subjective health complaints and modern health worries in patients with subjective food hypersensitivity. Dig Dis Sci. 2005;50:1245-1251.

16. Lillestøl K, Helgeland L, Lied GA, et al. Indications of "atopic bowel" in patients with self-reported food hypersensitivity. Aliment Pharmacol Ther. 2010;31:1112-1122.

17. Arslan G, Gilja OH, Lind R, Florvaag E, Berstad A. Response to intestinal provocation monitored by transabdominal ultrasound in patients with food hypersensitivity. Scand J Gastroenterol. 2005;40:386-394.

18. Kirwan JR, Hewlett S. Patient perspective: reasons and methods for measuring fatigue in rheumatoid arthritis. J Rheumatol. 2007;34: 1171-1173.

19. Fisk JD, Ritvo PG, Ross L, Haase DA, Marrie TJ, Schlech WF. Measuring the functional impact of fatigue: initial validation of the fatigue impact scale. Clin Infect Dis. 1994;18:Suppl 1:S79-S83.

20. Flensner G, Ek AC, Söderhamn O. Reliability and validity of the Swedish version of the Fatigue Impact Scale (FIS). Scand J Occup Ther. 2005;12:170-180.

21. Huet PM, Deslauriers J, Tran A, Faucher C, Charbonneau J. Impact of fatigue on the quality of life of patients with primary biliary cirrhosis. Am J Gastroenterol. 2000;95:760-767.

22. Mathiowetz V. Test-retest reliability and convergent validity of the Fatigue Impact Scale for persons with multiple sclerosis. Am J Occup Ther. 2003;57:389-395. 
23. Eriksen HR, Ihlebaek C, Ursin H. A scoring system for subjective health complaints (SHC). Scand J Public Health. 1999;1:63-72.

24. Kane SV, Sandborn WJ, Rufo PA, et al. Fecal lactoferrin is a sensitive and specific marker in identifying intestinal inflammation. $\mathrm{Am} \mathrm{J}$ Gastroenterol. 2003;98:1309-1314.

25. Longstreth GF, Thompson WG, Chey WD, Houghton LA, Mearin F, Spiller RC. Functional bowel disorders. Gastroenterology. 2006;130: 1480-1491.

26. Talley NJ, Verlinden M, Jones M. Validity of a new quality of life scale for functional dyspepsia: a United States multicenter trial of the Nepean Dyspepsia Index. Am J Gastroenterol. 1991;94:2390-2397.

27. Talley NJ, Verlinden M, Jones M. Quality of life in functional dyspepsia: responsiveness of the Nepean Dyspepsia Index and development of a new 10-items short form. Aliment Pharmacol Ther. 2001;15: 207-216.

28. Morken MH, Lind RA, Valeur J, Wilhelmsen I, Berstad A. Subjective health complaints and quality of life in patients with irritable bowel syndrome following Giardi lamblia infection: a case control study. Scand J Gastroenterol. 2009;44:308-313.

29. Wilhelmsen I, Mulindi S, Sankok D, Wilhelmsen AB, Eriksen HR, Ursin H. Subjective health complaints are more prevalent in Maasais than in Norwegians. Nord J Psychiatry. 2007;61:304-309.

30. Chronbach IJ, Shavelson RJ. My current thoughts on coefficient alpha and successor procedures. Educ Psychol Meas. 2004;64:391-418.

31. Bland JM, Altman DG. Validating scales and indexes. BMJ. 2002;324: 606-607.

32. Lied GA, Lillestøl K, Lind R, et al. Perceived food hypersensitivity: a review of 10 years of interdisciplinary research at a reference center. Scand J Gastroenterol. 2011;46:1169-1178.

33. Butt HL, Dunstan R, McGregor NR, Roberts TK. Bacterial colonosis in patients with persistent fatigue. Presented at the third international Alison Hunter Memorial Foundation clinical and scientific conference, December 1-2, 2001, Sydney, Australia.

34. Hassoun Z, Willems B, Deslauriers J, Nguyen BN, Huey PM. Assessment of fatigue in patients with chronic hepatitis $\mathrm{C}$ using the Fatigue Impact Scale. Dig Dis Sci. 2002;47:2674-2681.

35. Lind R, Lillestøl K, Valeur J, et al. Job stress and coping strategies in patients with subjective food hypersensitivity. Scand JPsychol. 2010;51: 179-184
36. Whitehead WE, Palsson O, Jones KR. Systematic review of the comorbidity of irritable bowel syndrome with other disorders: what are the causes and implications? Gastroenterology. 2002;122: $1140-1156$.

37. Hillilä MT, Färkkila NJ, Färkkila MA. Societal costs for irritable bowel syndrome - a population based study. Scand J Gastroenterol. 2010;45:582-591.

38. Heaton KW, Ghosh S, Bradden FE. How bad are the symptoms and bowel dysfunction of patients with the irritable bowel syndrome? A prospective, controlled study with emphasis on stool form. Gut. 1991;32:73-79.

39. van der Werf SP, Prins JB, Vercoulen JH, van der Meer JW, Bleijenberg G. Identifying physical activity patterns in chronic fatigue syndrome using actigraphic assessment. J Psychosom Res. 2000;49: 373-379.

40. Shepherd SJ, Lomer MC, Gibson PR. Short-chain carbohydrates and functional gastrointestinal disorders. Am J Gastroenterol. 2013;108:707-717.

41. Valeur J, Morken MH, Norin E, Midtvedt T, Berstad A. Carbohydrate intolerance in patients with self-reported food hypersensitivity: Comparison of lactulose and glucose. Scand J Gastroenterol. 2009;44: 1416-1423.

42. Winwood PC, Winefield AH, Lushington K. Work-related fatigue and recovery: the contribution of age, domestic responsibilities and shiftwork. $J$ Adv Nurs. 2006;56:438-449.

43. Nagai M, Morikawa Y, Kitaoka K, et al. Effects of fatigue on immune function in nurses performing shift work. J Occup Health. 2011;53: 312-319.

44. Choi BC, Pak AW. A catalog of biases in questionnaires. Public Health Research, Practice and Policy. 2005;20:1-13.

45. DavidA, Pelosi A, McDonald E, et al. Tired, weak, or in need of rest: fatigue among general practice attendees. BMJ. 1990;301:1199-1202.

46. Lee KJ, Tack J. Altered intestinal microbiota in irritable bowel syndrome. Neurogastroenterol Motil. 2010;22:493-498.

47. Zhou QQ, Zhang B, Verne GN. Intestinal membrane permeability and hypersensitivity in the irritable bowel syndrome. Pain. 2009;146: $41-46$
Clinical and Experimental Gastroenterology

\section{Publish your work in this journal}

Clinical and Experimental Gastroenterology is an international, peerreviewed, open access journal, publishing all aspects of gastroenterology in the clinic and laboratory, including: Pathology, pathophysiology of gastrointestinal disease; Investigation and treatment of gastointestinal disease; Pharmacology of drugs used in the alimentary tract;

\section{Dovepress}

Immunology/genetics/genomics related to gastrointestinal disease. This journal is indexed on CAS. The manuscript management system is completely online and includes a very quick and fair peer-review system. Visit http://www.dovepress.com/testimonials.php to read real quotes from published authors. 